\title{
Initial Experience With the Retrosigmoid Inframeatal Approach in Skull Base Surgery
}

\author{
Madjid Samii ${ }^{1,2}$; Maysam Alimohamadi ${ }^{1,2,} ;$ Hussam Metwali ${ }^{1}$; Venelin Gerganov ${ }^{1}$

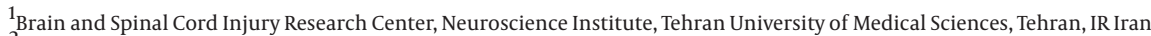 \\ ${ }^{2}$ International Neuroscience Institute, Hannover, Germany \\ *Corresponding author. Maysam Alimohamadi, International Neuroscience Institute, Hannover, Germany. Tel: +49-51127092700, Fax: +49-51127092706, E-mail: alimohamadi59@gmail.com
}

Received: May 4, 2014; Accepted: December 28, 2014

\begin{abstract}
Introduction: Lesions of the petrous apex involving the inframeatal/infralabyrinthine region are challenging for surgical removal and have a high risk of approach related complications. Traditionally, various extensive skull-base approaches have been used for these lesions. The aim of this study was to present and evaluate our initial experience with a new hearing-preserving extension of the classic retrosigmoid approach to the inframeatal/infralabyrinthine area.

Case Presentation: The approach was used in four patients harboring lesions in the petrous apex with variable extension in the inframeatal/infralabyrinthine region. The surgical accessibility of the lesions offered by the approach, completeness of tumor removal, the outcome, in particular, the functional outcome and complication rate, were assessed.

Conclusions: The tumor could be gross totally resected in all the four cases. No approach-related morbidities occurred. Serviceable hearing and normal facial nerve functions were preserved in all cases. Our initial experience with the retrosigmoid inframeatal approach revealed that it provides sufficient access to the inframeatal/infralabyrinthine region and offers the possibility of complete tumor removal without any major complications. It allows for hearing and facial nerve functional preservation.
\end{abstract}

Keywords: Pterous Bone; Jugulare Tumors; Skull Base; Posterior Fossa Tumor

\section{Introduction}

The inframeatal region extends from the lower boundary of the internal auditory canal to the exit zone of the glossopharyngeal nerve and is bounded anteriorly by the petrous internal carotid artery and medially by the petrous apex. It is involved by lesions, either primarily (chordoma, chondrosarcoma, cholesterol granuloma) or extension of tumors from neighboring structures (schwannoma, meningioma, paraganglioma, etc.) (1-5).

Difficulty of direct surgical access due to proximity to the midline and deep-seated location, along with the vitality of adjacent structures such as acousticofacial bundle, lower cranial nerve and jugular bulb, encouraged skull base surgeons to search for appropriate approaches to the lesions of inframeatal petrous apex.

Extensive approaches such as variable posterior transpetrosal approaches, peresigmoidal approach, Fisch infratemporal approaches and far lateral suprajugular approach have been shown to have high rate of approach related morbidities, particularly hearing loss, facial nerve paresis, venous damage and postoperative CSF fistulae $(1,6-14)$

In this article, we aimed to present an evolution of the worldwide familiar standard retrosigmoid approach to address the pathologies of inframeatal region, to mini- mize approach related complications and improve completeness of tumor removal and functional outcome of patients.

\section{Case Presentation}

\subsection{Positioning and Skin Preparation}

The operation was performed in the semi-sitting position. The head was flexed and rotated to the ipsilateral side. Positioning was performed under monitoring of the somatosensory evoked potentials (SSEP). A 3-cm strip of hair was shaved behind the ear. The mastoid tip, the digastric groove and the line connecting the zygoma to the inion were marked on the skin. A C shaped skin incision was marked 2 finger breadth behind the digastric groove extending inferiorly below the level of the mastoid tip and above the zygoma-inion line.

\subsection{Skin Incision and Craniotomy}

After skin incision, an autologous subcutaneous fat graft was taken to be used for air cells packing. The muscles were incised in layer. The suboccipital craniotomy

Copyright (C) 2015, Tehran University of Medical Sciences. This is an open-access article distributed under the terms of the Creative Commons Attribution-NonCommercial 4.0 International License (http://creativecommons.org/licenses/by-nc/4.0/) which permits copy and redistribute the material just in noncommercial usages, provided the original work is properly cited. 
was performed. Opening the foramen magnum was not required, but the lateral edge of the sigmoid sinus, the sinus knee and the lower margin of the transverse sinus should be exposed. The dura was incised a few millimeters posterior to the sigmoid sinus and then posteriorly extended inferior to the transverse sinus.

\subsection{Intradural Dissection}

It starts with CSF release from the lateral cerebellomedullary cistern. The arachnoid was dissected exposing the lower cranial nerves and the VII/VIII cranial nerves complex. Cerebellar retraction was performed carefully under control of the auditory evoked potentials.

\subsection{Identification of the Entry Point}

The entry point for drilling was located below the lower border of the porus acusticus and above the dural exit of the glossopharyngeal nerve. Before starting drilling, the arachnoid of the lower cranial nerves as well as the VII/VIII cranial nerves complex should be completely dissected.

\subsection{Inframeatal Drilling and Tumor Resection}

The dura inferior to the porus and superior to the dural exit of the lower cranial nerves were excised exposing the inframeatal part of the temporal bone. A high speed drill was used to drill the inframeatal ridge. Drilling in this area allows access to the infralabyrinthine part of the temporal bone anteriorly till the level of the horizontal segment of the internal carotid artery. Drilling medially and superiorly along the posterior petrous dura and the inferior petrosal sinus allows access to the petrous apex. The tumor was resected with usual microsurgical techniques. The operative endoscope can be applied for inspection of the tumor bed, confirmation of tumor resection and removal of tumor remnants.

\subsection{Closure}

After meticulous hemostasis, the drilled area was packed with autologous fat graft and fixed with fibrin glue. The dura was closed water tightly. The mastoid air cells were packed with autologous fat graft and fibrin glue. The craniectomy site was reconstructed with artificial bone substitute. The wound was closed in layers.

\subsection{Approach Accessibility and Limitation}

The retrosigmoid intradural inframeatal approach can provide access to the inframeatal/ infralabyrinthine region and the inferior part of the petrous apex. This corridor is extending from the posterior surface of the petrous bone inferior to the internal auditory canal posteriorly to the horizontal segment of the internal carotid artery anteriorly. Medially, the corridor includes the infe- rior part of the petrous apex. This approach alone cannot provide access to the middle fossa dura or the Meckel's cave. To access the Meckel's cave, the approach should be combined with retrosigmoid intradural suprameatal approach. While performing this approach, there is no clear anatomical landmark of the horizontal segment of the petrous internal carotid artery. We used the neuronavigator and microdoppler probe to localize the internal carotid artery. The neuroendoscopy with different angles is a very helpful tool for inspection of the tumor bed and allows further endoscope assisted tumor removal. It provides visualization in many angles and discovering and removal of any remaining tumor.

\subsection{Illustrative Case}

A thirty-one-year-old male patient presented with progressive swallowing difficulties as well as headache. MRI was performed showing contrast enhancing lesion expanding the jugular foramen and the jugular fossa with small extracranial extension through the jugular foramen (Figure $1 \mathrm{~A}$ and $1 \mathrm{~B}$ ). The retrosigmoid inframeatal approach was chosen to resect the tumor. A retrosigmoid craniectomy was performed. The seventh and eights cranial nerves were exposed (Figure $2 \mathrm{~A}$ ). The drilling was started below the internal auditory canal and the VII/VIII complex (1) as shown in the Figure $2 \mathrm{~B}$ and $2 \mathrm{C}$. The tumor (2) was further resected between the VII/VIII (1) and the lower cranial nerves (shown by number 3 in the Figure $2 \mathrm{D}$ ). The intratemporal part of the tumor was resected (Figure $2 \mathrm{E}$ ) and the resection of the tumor was confirmed using the operative endoscope. The anterior boundary of resection was the internal carotid artery (shown by number 5 in the Figure 2 F). The postoperative images confirmed resection of the tumor (Figure 1C and 2D).

\section{Discussion}

The inframeatal ridge of bone is bounded by the internal auditory canal superiorly and the dural sleeve of the glossopharyngeal nerve inferiorly. This part of the temporal bone is devoid of important structures. Drilling of this bone provides an access to the area inferiorly and anteromedially to the internal auditory canal, the infralabyrinthine region, lower petroclival fissure and the petrous apex. The infralabyrinthine region of the temporal bone is bounded by the labyrinth and internal auditory canal superiorly, the jugular bulb and the inferior petrosal sinus inferiorly, the internal carotid artery anteriorly and the dura covering the posterior surface of the petrous bone posteriorly. Anteromedially, this space is continuous with the petrous apex.

Lateral transtemporal approaches are mainly used by ENT (Ear, Nose and Throat) surgeons. The transcochlear approach sacrifices hearing and requires transposition of facial nerves with the possibility of facial nerve palsy (15). 

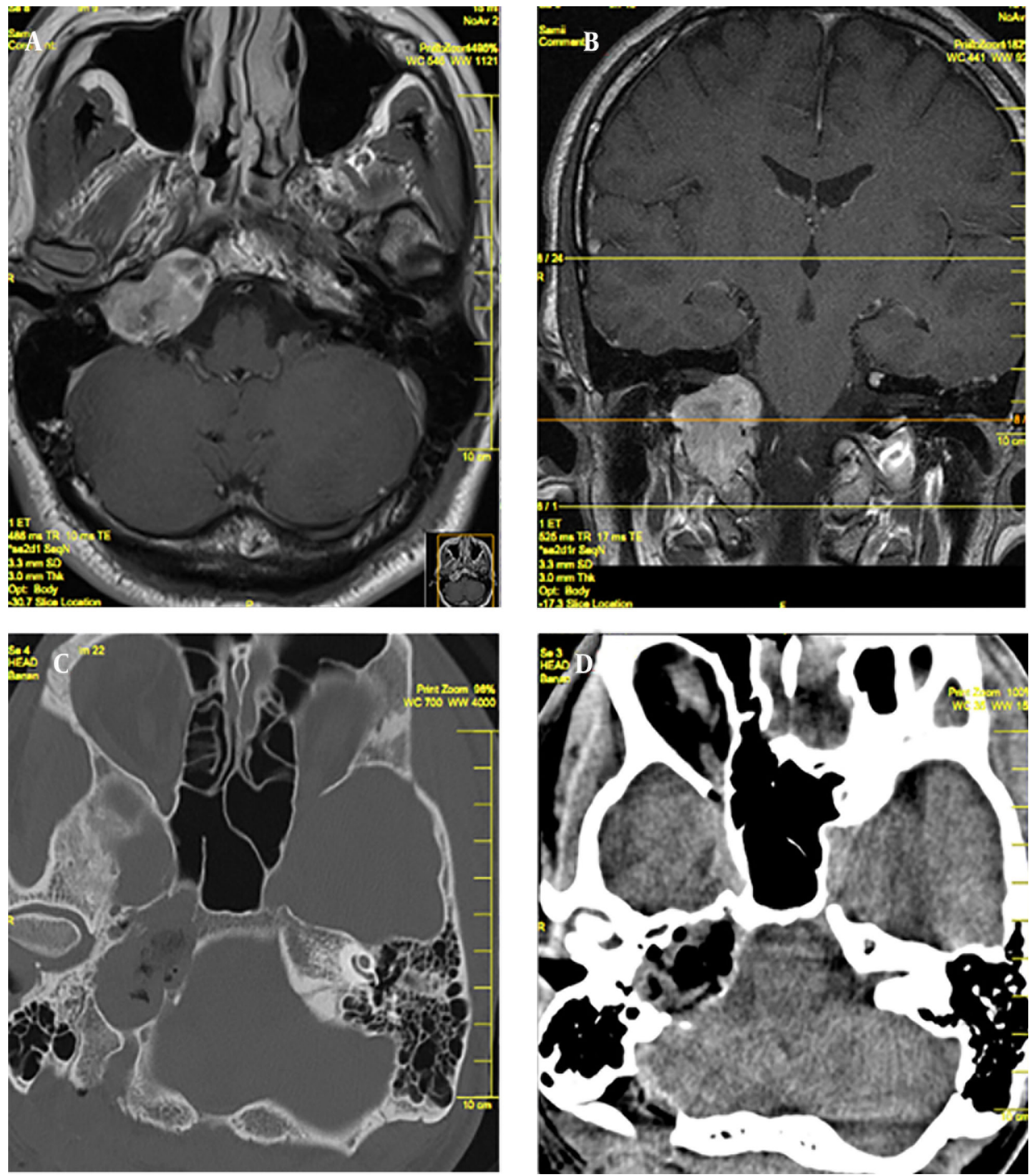

Figure 1. Axial and Coronal Preoperative MRI With Contrast of the Patient (A, B) and Postoperative CT Scan (C, D)

The infratemporal fossa approach type A is probably the most popular approach among ENT surgeons. Despite the outstanding work of Fisch and others, there are inherent risks of morbidities in the surgical approach, especially facial nerve palsy and conductive hearing loss (16-18). More recently, the presigmoid infralabyrinthine approach has been discussed by some authors for pathologies of inframeatal petrous region $(16,17,19)$. 
Figure 2. Stepwise Tumor Resection of the Illustrative Case
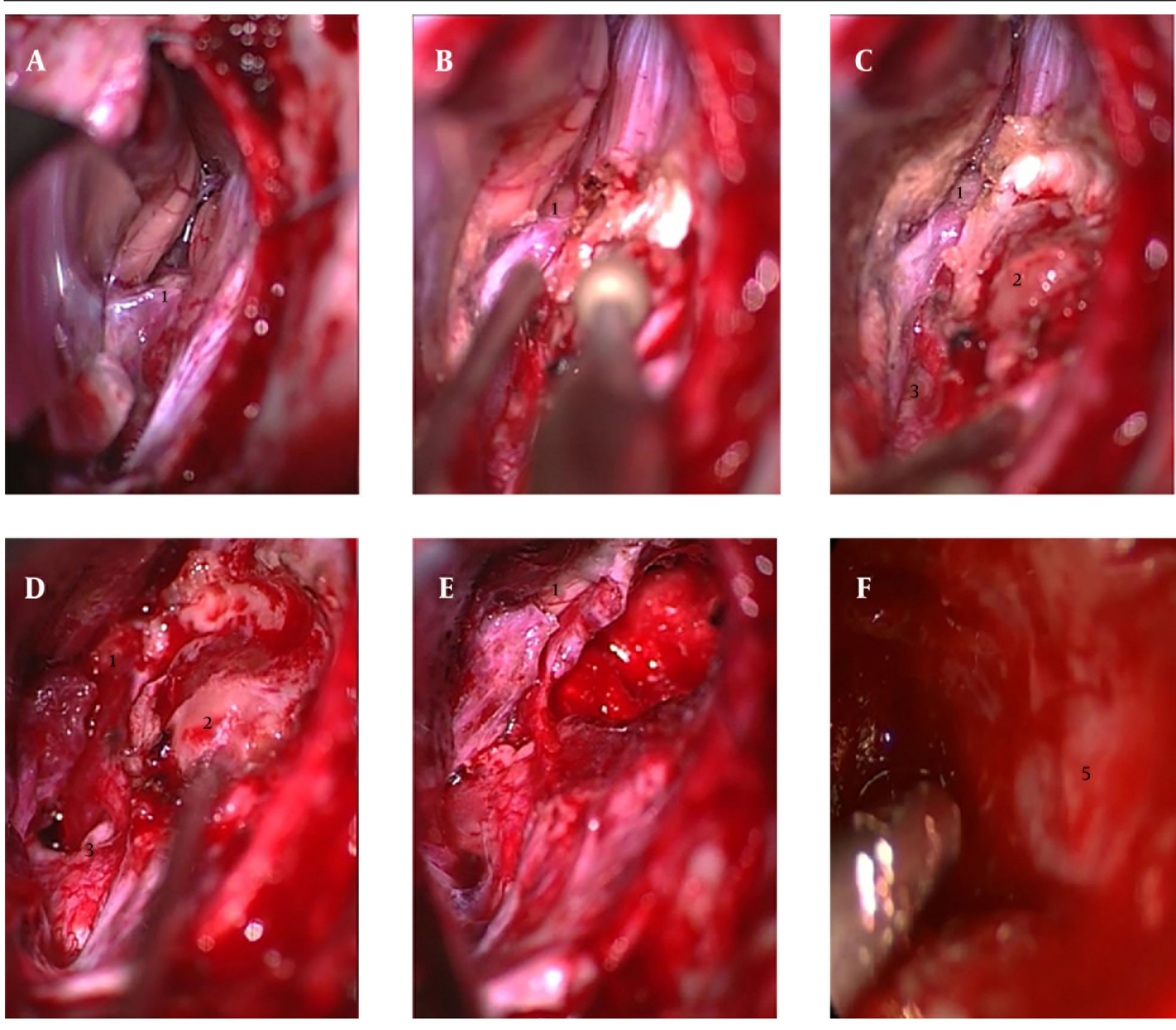

1, VII/VIII complex; 2, Tumor; 3, Lower cranial nerves; 5, internal carotid artery.

This approach also needs skeletonizing the sigmoid sinus and extensive drilling of mastoid and fallopian canal to expose the facial nerve, even though facial nerve rerouting is not mandatory. This approach has a limited exposure of the intradural and especially the cisternal parts of the lesion, and the brainstem and cranial nerves usually come into view only after removal of a major bulk of tumor.

Another alternative is the endoscopic endonasal approaches, which are technically difficult because of lateral extension of these lesions and the high risk of abducent nerve injuries (14).

Hereby, we described our initial experience with inframeatal extension of the retrosigmoid approach. In this approach, we tried to balance adequate exposure with minimal interference with neurovascular structures. The approach was applied in four cases with differ- ent pathologies, but sharing a common feature of invasion of the inframeatal/infralabyrinthine/apical region of the petrous bone.

Preoperative analysis of surgical anatomy of lesion and nearby structures in preoperative imaging is mandatory to detect possible deviation from the normal anatomy, particularly a high located jugular bulb, which can impede the surgical approach (20).

In this approach, it is possible for the surgeon to have a view of the brainstem and exit zones of the cranial nerves early in the course of operation and after removal of inframeatal ridge it. Using a neuronavigator and Doppler, tailored to the extension of the pathology, the surgeon can identify the surrounding vital structures for a more radical tumor removal. The neuroendoscopy has a complementary role and can confirm the completeness of tumor resection $(21,22)$. 
In this small series, there were no approach-related complications. Importantly, the functional outcome regarding facial nerve, hearing and lower cranial nerves, was satisfactory with no new deficits. The preoperatively planned amount of tumor resection from the target could be achieved. Therefore, we consider the hearing and facial nerve-preserving retrosigmoid inframeatal approach to the inframeatal/infralabyrinthine apical region of the petrous bone as an acceptable alternative to other approaches to this region.

\section{References}

1. Rhoton AJ. The temporal bone and transtemporal approaches. Neurosurgery. 2000;47(3 Suppl):S211-65.

2. Sanna M, Pandya Y, Mancini F, Sequino G, Piccirillo E. Petrous bone cholesteatoma: classification, management and review of the literature. Audiol Neurootol. 2011;16(2):124-36.

3. Aubry K, Kovac L, Sauvaget E, Tran Ba Huy P, Herman P. Our experience in the management of petrous bone cholesteatoma. Skull Base. 2010;20(3):163-7.

4. Black B, Gutteridge I. Acquired cholesteatoma: classification and outcomes. Otol Neurotol.2011;32(6):992-5.

5. Bambakidis NC, Kakarla UK, Kim LJ, Nakaji P, Porter RW, Daspit $\mathrm{CP}$, et al. Evolution of surgical approaches in the treatment of petroclival meningiomas: a retrospective review. Neurosurgery. 2007;61(5 Suppl 2):202-9.

6. Sakata K, Al-Mefty O, Yamamoto I. Venous consideration in petrosal approach: microsurgical anatomy of the temporal bridging vein. Neurosurgery. 2000;47(1):153-60.

7. Morisako H, Goto T, Nagata T, Chokyu I, Ichinose T, Ishibashi K, et al. Middle skull base approach with posterolateral mobilization of the geniculate ganglion to access the clival regions. Neurosurgery. 2011;69:ons88-94.

8. Goel A. Middle fossa sub-Gasserian ganglion approach to clivus chordomas. Acta Neurochir. 1995;136(3-4):212-6.

9. Day JD. The middle fossa approach and extended middle fossa approach: technique and operative nuances. Neurosurgery. 2012;70:ons192-201.

10. De la Cruz A, Teufert KB. Transcochlear approach to cerebellopontine angle and clivus lesions: indications, results, and complications. Otol Neurotol. 2009;30(3):373-80.

11. Avci E, Dagtekin A, Akture E, Uluc K, Baskaya MK. Microsurgical anatomy of the vein of Labbe. Surg Radiol Anat. 2011;33(7):569-73.

12. Samii M, Tatagiba M, Carvalho GA. Retrosigmoid intradural suprameatal approach to Meckel's cave and the middle fossa: surgical technique and outcome. J Neurosurg. 2000;92(2):235-41.

13. Guppy KH, Origitano TC, Reichman OH, Segal S. Venous drainage of the inferolateral temporal lobe in relationship to transtemporal/transtentorial approaches to the cranial base. Neurosurgery. 1997;41(3):615-9.

14. Barges-Coll J, Fernandez-Miranda JC, Prevedello DM, Gardner P, Morera V, Madhok R, et al. Avoiding injury to the abducens nerve during expanded endonasal endoscopic surgery: anatomic and clinical case studies. Neurosurgery. 2010;67(1):144-54

15. Sanna M, Mazzoni A, Saleh EA, Taibah AK, Russo A. Lateral approaches to the median skull base through the petrous bone: the system of the modified transcochlear approach. J Laryngology Otol. 2007;108(12).

16. Cinibulak Z, Krauss JK, Nakamura M. Navigated minimally invasive presigmoidal suprabulbar infralabyrinthine approach to the jugular foramen without rerouting of the facial nerve. $\mathrm{Neu}$ rosurg. 2013;73:ons3-15.

17. Borba LA, Ale-Bark S, London C. Surgical treatment of glomus jugulare tumors without rerouting of the facial nerve: an infralabyrinthine approach. Neurosurg Focus. 2004;17(2):E8.

18. Fisch U, Fagan P, Valavanis A. The infratemporal fossa approach for the lateral skull base. Otolaryngol Clin North Am.1984;17(3):513-52.

19. Gjuric M, Bilic M. Transmastoid-Infralabyrinthine Tailored Surgery of Jugular Paragangliomas. Skull Base. 2009;19(01):75-82.

20. Jacob CE, Rupa V. Infralabyrinthine approach to the petrous apex. Clin Anat. 2005;18(6):423-7.

21. Samii M, Gerganov VM. Surgery of Extra-Axial Tumors of the Cerebral Base. Neurosurg. 2008;62(Supplement 3):1153-68.

22. Samii M, Metwali H, Samii A, Gerganov V. Retrosigmoid intradural inframeatal approach: indications and technique. Neurosurg. 2013;73:ons53-60. 